\title{
FACTORES CRÍTICOS DE FORMACIÓN TECNOLÓGICA EN LAS UNIVERSIDADES ACREDITADAS DE EL SALVADOR
}

Por: José Francisco Guzmán Rivera

L a educación tecnológica es aquella vertiente de la educación que promueve instancias de aprendizaje, permite a los estudiantes una base de conocimientos, habilidades y actitudes (competencias cognitivas) para desenvolverse adecuadamente como creadores, elaboradores y usuarios críticos de la tecnología (competencias productivas), preparándolos para ser actores del desarrollo que manejan la tecnología al servicio de las necesidades humanas (competencias sociales).

Esta área del sistema educativo tiene por objeto, despertar en los estudiantes, la toma de conciencia de la creciente importancia y presencia del mundo, desarrollar la capacidad operativa que les permita, como ciudadanos, participar en su evolución y control, lo que implica reflexionar críticamente acerca de los problemas del mundo productivo, manejar los conocimientos y habilidades que les posibiliten desenvolverse con idoneidad, solvencia, responsabilidad y creatividad al afrontar problemas o circunstancias, buscando colaborar en mejorar la calidad de vida de la sociedad.

La educación tecnológica procura promover en los estudiantes una actitud científica al enfrentar problemas vinculados a la tecnología y una disposición a aplicar el método científico en la resolución de los mismos, destacando la responsabilidad del hombre y su accionar tecnológico, frente a la sociedad y al mundo, teniendo en cuenta el impacto $y$ las consecuencias de este accionar en ambos campos; por tanto, busca desarrollar no sólo capacidades de ejecución manual e intelectual, sino también la capacidad creativa, entendiendo que el actual nivel de desarrollo tecnológico así lo exige.

Esta vertiente de la educación, promueve la cultura tecnológica, actor clave del desarrollo económico, social y cultural de un país; académicamente, se basa en el valor educativo de tecnología teniendo en cuenta que es tan importante la teoría como la práctica.

Es decir, el saber hacer como el hacer para saber; considerando el valor formativo y cultural que puede llegar a tener el trabajo manual cuando se le enfoca como solución de problemas vinculados al acontecer cotidiano, el hacer se asume como elemento didáctico; el hacer mantiene despierta la atención y la curiosidad de los estudiantes y posibilita una participación activa, durante el proceso de aprendizaje, pues no sólo el docente interviene activamente en este, sino también los estudiantes, todo esto dinamiza dicho proceso y bien orientado logra que los estudiantes se muevan dentro del campo de la técnica con la mentalidad de un investigador.
La tecnología, como saber sistematizado, tiene valor académico porque su intencionalidad es integrar el mundo del saber teórico con el de la práctica; ayuda a comprender la realidad desde la unidad teoría-práctica, es parte de la función que tiene la educación tecnológica.

En función de lo planteado, es clara la importancia de la educación tecnológica y la necesidad de incorporarla al currículo universitario, porque se convierte en uno de los ejes integradores del conocimiento y en pilar del rol social de la academia; la noción de esta educación proviene de un concepto amplio, capaz de cubrir las etapas formativas construidas en los procesos básicos de la capacitación humana, pero privilegiando las vertientes del trabajo, del conocimiento y de la innovación tecnológica.

Sin embargo, en una investigación realizada por la Universidad Tecnológica en el 2004 sobre la situación actual de la educación tecnológica de las universidades acreditadas de El Salvador, basada en la existencia de componentes tecnológicos directos e indirectos que exige esta vertiente de la educación entre ellos: infraestructura tecnológicamente adecuada, campos experimentales, programas métodos y contenidos correspondientes a la formación en estudio, docentes tecnológicamente formados, estudiantes a tiempo completo debido a la exigencia y rigurosidad de este tipo de educación, prácticas formativas y de componentes indirectos como: intercambios académicos al extranjero de docentes y estudiantes, articulación curricular entre universidades, vínculos universidad empresa.

Entre los resultado se encontró la siguiente situación que obstaculiza el desarrollo de una adecuada formación tecnológica en primer orden: la situación de ausencia, escasez y sub utilización de componentes tecnológicos que presentan algunas universidades acreditadas, no están en función directamente proporcional con la educación tecnológica, es decir, que ante el inadecuado funcionamiento de cada uno de ellos no contribuye a aumentar la calidad de la formación en los estudiantes; debido a que ante la falla y ausencia de uno o varios de los componentes analizados repercute negativamente sobre la formación del estudiante, ya que la falta de vinculación y contacto por medio de la práctica no garantiza el desarrollo de competencias; situación de ausencia y escasez que obstruye la implementación y aplicación de esa vertiente de la educación.

Los hallazgos encontrados en la investigación presentan los siguientes problemas para el desarrollo de una adecuada y eficiente formación tecnológica: $57.14 \%$ de las universidades no tiene una infraestructura tecnológicamente adecuada que le permita tener 
un amplio campo de acción en sus respectivas áreas, concernientes a la educación técnico-práctica y a la investigación; en una proporción mayor la posesión de campos experimentales con $71.43 \%$. Por tanto, como componentes tecnológicos, son vitales para la práctica y desarrollo de conocimientos y habilidades, ya que permiten su ampliación por medio de la experimentación y aplicación; sin embargo, la situación en que se encuentran, refleja la deficiencia obtenida a la hora de formar la capacidad científica y tecnológica de los estudiantes.

$71.43 \%$ de las universidades, no están en línea con la práctica tecnológica, es decir, que los currículos, programas, métodos y contenidos, no tienen la suficiente idoneidad o no corresponden a las calificaciones requeridas en el proceso de enseñanza aprendizaje, no satisfacen las exigencias de la formación tecnológica, por tanto según lo manifestado por algunos funcionarios y docentes entrevistados, es necesario modificarlos y adaptarlos, ya que son más teóricos que prácticos.

En la mayoría de universidades, el cuerpo docente es de tiempo parcial, por tanto no corresponde a las exigencias de la educación tecnológica, ya que esta exige que para una formación tecnológica sea eficiente, el docente tiene que tener el tiempo disponible para fortalecer el conocimiento, el dominio de tecnologías y de esta forma transferirlas al estudiante.

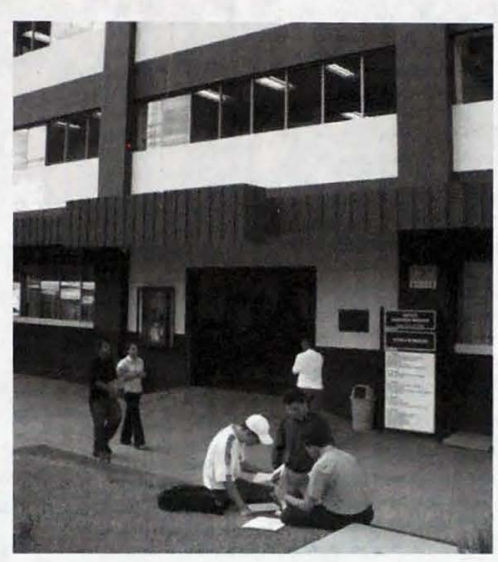

Ante esta situación temporal en el proceso de enseñanza-aprendizaje por parte de los docentes, no es de extrañar, sostiene la investigación, que la mayoría de ellos solo posea una preparación básica según la rama especifica; por tanto, no se logra acumular y desarrollar el conocimiento científico y la transmisión de este a los estudiantes y contribuir a la formación requerida.

$57.14 \%$ de las universidades, sostiene que sus estudiantes dejan de realizar prácticas formativas ya que la mayoría de éstos trabaja y la disponibilidad en el tiempo es un obstáculo para la formación tecnológica; por tanto, el perfil del estudiante en la mayoría de las universidades es de tiempo parcial debido a sus compromisos económicos y laborales; esto no permite que se desarrollen tecnológicamente, ya que no hay una aplicación de procedimientos organizados en el aprendizaje.

Unido a la situación anterior, $71.14 \%$ de las universidades manifiesta que no existen intercambios académicos y que debido a esa situación no se contribuye a aumentar la capacidad, conocimiento y habilidades de los docentes y estudiantes; es decir, que no se fortalece la calidad técnico- académica, a fin que los educandos puedan crear, aplicar, diseńar, perfeccionar y aprovechar en forma óptima los programas de desarrollo tecnológico provenientes de países desarrollados, enfatizando el desarrollo especializado de los docentes.

Las siete universidades analizadas, sostienen que no existe articulación curricular entre ellas y que en lugar de compartir recursos, conocimientos y experiencias, solo dedican sus potencialidades a rivalizar y competir por la absorción de la demanda estudiantil. Asimismo, $85.71 \%$ expresan que no existen vínculos con los sectores empresariales.

Con los resultados obtenidos por el estudio en que la mayoría de universidades presentan deficiencias y rompen con la virtud que tienen cada uno de los componentes en la formación tecnológica, se deduce que en algunas universidades, existen carreras que solo poseen el nombre de tecnológicas, porque no forman y desarrollan "tecnológicamente" al estudiante, dada la ausencia, escasez y sub utilización de componentes y recursos requeridos para esta actividad educativa. De esta forma, según el contexto actual, las universidades que cuentan con algunos recursos tecnológicos, pero que son escasos en otros, la formación tecnológica recibida en ellas no es lo suficientemente capaz para que sus egresados y graduados puedan competir en mercados modernos que el mundo actual posee. Por tanto cabe preguntar: ¿Qué tipo de formación tecnológica recibe un estudiante de ingeniería civil, agronomía o arquitectura sin laboratorios y campos experimentales? ¿Cuál es la visión de ciencia y tecnología que posee la mayoría de universidades acreditadas? ¿Cuáles son las esperanzas de desarrollo tecnológico para el país ante esta situación?

Para comprender mejor, se explica en dicho estudio a manera de ejemplo, que pasa con la formación tecnológica de los estudiantes en algunas carreras como el caso de la arquitectura, esta la sirven 6 de 7 universidades y para el proceso de enseñanza aprendizaje (prácticas formativas de campo) se requiere el uso de campos experimentales, equipo tecnológico, aplicación teórica y práctica en laboratorios tecnológicamente adecuados. Donde el $71.43 \%$ de las universidades presentan dificultades en estos componentes tecnológicos y las que lo poseen subutilizan este recurso. 
Ante esta situación el estudiante no logra desarrollar sus etapas de formación y con ellas las habilidades teóricas y prácticas, el desarrollo de fundamentación conceptual, manejo de métodos de trabajo; dominio instrumental en la que aprende los recursos técnicos y científicos para manejar proyectos arquitectónicos; dominio operativo en la que demuestran su capacidad de manejo de personal y de los proyectos con independencia; debido a que no hay una relación directa de este con los componentes tecnológicos y de esta forma no logra el crecimiento de una visión científica y técnica de su carrera.

Este mismo análisis y situación se explica para el caso de la carrera de ingeniería civil la cual la imparten 4 de 7 universidades, carrera que en el proceso de enseñanza-aprendizaje requiere el uso de laboratorios, trabajos de investigación, elaboración de proyectos y visitas de campo.

En este mismo orden de uso de componentes tecnológicos están entre otros las carreras siguientes: la ingeniería química, que proporciona bases científico-tecnológicas para el desarrollo y aplicación de los procesos de producción de bienes y servicios; la ingeniería agronómica la cual utiliza laboratorios debidamente equipados, plantas piloto, observación científica en áreas como fitotecnia, protección vegetal, química agrícola, suelos e ingeniería agrícola.

El problema está con la ingeniería industrial, esta especialidad la imparten la mayoría de universidades en estudio, tiene por finalidad investigar, proyectar, instalar, operar y mejorar sistemas de producción de bienes y servicios; se hace uso de laboratorios, discusión de problemas, trabajos de investigación, visitas técnicas a empresas; lo que sucede con esta, es que su campo experimental además de exigir laboratorios tecnológicamente adecuados, la constituyen fundamentalmente los vínculos empresariales y por medio de estos las prácticas formativas y como se detalla, los vínculos universidad-empresa no existen en 6 de 7 universidades; por tanto, impacta negativamente en la formación tecnológica de los estudiantes.

Es decir, que no se logra compartir, aprovechar, transferir y desarrollar experiencias técnico científicas que promuevan la investigación científica y la transferencia tecnológica. De la misma forma no se promueven planes o programas educativos acordes a la realidad nacional y al desarrollo científico-tecnológico de la región.

Al ubicar en el mismo nivel de análisis la situación de las otras ingenierías podemos decir, que también enfrentan iguales o peores dificultades para su desarrollo en el proceso de enseñanza aprendizaje. Esta misma problemática se repite o recae sobre los doctorados, maestrías y licenciaturas que están o dicen estar en línea con la educación tecnológica.

Frente a esta situación de ausencia, escasez y sub utilización de
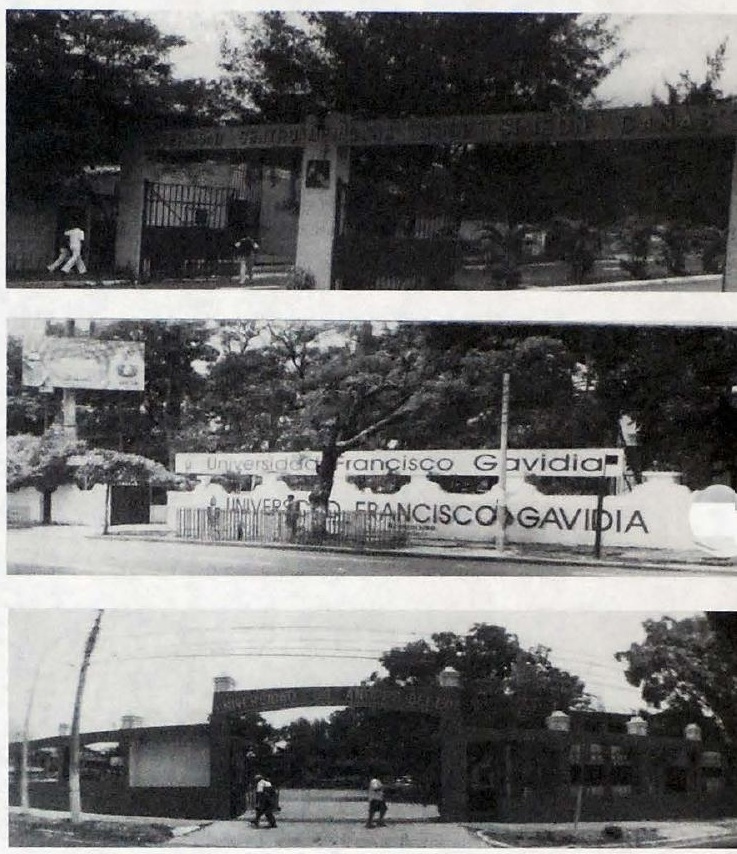

componentes y dispositivos tecnológicos que presenta la mayoría de universidades acreditadas se concreta lo siguiente: que la educación tecnológica que implementa (o dice implementar), la mayoría de las universidades acreditadas, presenta fuertes deficiencias para el manejo y formación de conocimientos y habilidades que le posibilite a los estudiantes desenvolverse con idoneidad y creatividad al momento de enfrentar problemas de carácter tecnológico, más que todo, en el área productiva; en la capacidad de plantear alternativas; en la promoción de una actitud cientifica al enfrentar problemas vinculados a la tecnología y a una disposición en aplicar el método científico en la resolución de los mismos.

Estas deficiencias también pueden notarse en el desarrollo no sólo de capacidades de ejecución manual e intelectual, sino también en la capacidad creativa, entendiendo que el actual nivel de desarrollo tecnológico así lo exige.

Se detalla que esta situación es debida, en parte, a la falta de preocupación y de rigurosidad académica en la aplicación de esta vertiente de la educación por parte de algunas universidades. No se promueve la cultura tecnológica, como actor clave del desarrollo económico, social y cultural de todo país. 
